\title{
Modification of Diurnal Changes in the Ultrastructure of Synaptic Ribbons of the Turtle
}

\author{
Hiroshi Abe and Toshi Yuki Yamamoto \\ Department of Anatomy, Tohoku University School of \\ Medicine, Sendai 980
}

\begin{abstract}
ABE, H. and Yamamoto, T.Y. Modification of Diurnal Changes in the Ultrastructure of Synaptic Ribbons of the Turtle. Tohoku J. exp. Med., 1988, 156 (4), 381-393 — Synaptic ribbons of rod cells of the red-eared turtle under light-dark (LD) cycle, continuous dark (DD) and continuous light (LL) were examined by electron microscopy. In the LD cycle, several stick-shaped (plateshaped, three-dimensionally) ribbons formed multilayered ribbon complexes at $1000 \mathrm{hr}$, and they turned into aggregates of rounded ribbons at $1900 \mathrm{hr}$ ( $1 \mathrm{hr}$ after light-off). In DD, stick-shaped ribbons were situated singly near presynaptic membranes. Large ribbon complexes consisting of 7-.8 parallel layers, as shown in the LD cycle, were not observed. Aggregates of rounded ribbons, or club-shaped ones could not be observed. In LL, aggregates of irregular ribbon fragments were noted. None of the ribbons in this regimen showed sharp contours. Cyclic LD illumination is necessary to form multilayered ribbon complexes and rounded ribbons. Internal circadian rhythm contributes little part to the diurnal changes in the structure of the synaptic ribbons. —_ synaptic ribbons; turtle photoreceptor cells; diurnal changes; electron microscopy
\end{abstract}

Synaptic ribbons are presynaptic organelles in the photoreceptor cells (Sjöstrand 1958) and pineal cells of many vertebrate species (Vollrath 1981). Studies on the postnatal development suggest that the synaptic ribbons are functionally related to the sense of vision (Olney 1968; McArdle et al. 1977). According to their reports, the ribbons increase their frequency after birth. They may play an important role in synaptic transmission between receptor cells and the second order neurons.

In adult animals, synaptic ribbons had been recognized as contant structures. But recently, quantitative morphological studies suggested that the frequency and length of ribbons in the photoreceptor cells and pineal cells are affected by lighting conditions (Wagner 1973, 1975; Spadaro et al. 1978; McNulty 1981). We reported that, in the turtle retina, dynamic diurnal changes take place in morphology of the ribbons in rod cells (Abe and Yamamoto 1984). The synaptic ribbons grow into multilayered complexes composed of several layers of stickshaped ribbon profiles and associated vesicles from midnight to noon. They are

Received October 12,1988; revision accepted for publication November 11, 1988. 
disintegrated into irregular fragments from noon to evening, and show rounded profiles at $1 \mathrm{hr}$ after light-off.

Are these diurnal changes induced only by lighting conditions, or are they under the control of internal circadian rhythm as suggested in the ribbons of the pineal organ (McNulty 1981; Matsushima et al. 1983)? This study was undertaken to analyze the mechanism of diurnal changes of synaptic ribbons in rod cells of the turtle by modifying the lighting condition. These diurnal changes will be considered to be induced by internal circadian rhythm, if various profiles of synaptic ribbons in light-dark cycle (i.e., multilayered ribbon complexes, aggregates of irregular ribbons, and aggregates of rounded ribbons) (Abe and Yamamoto 1984) can be observed even in continuous darkness. On the contrary, if the formation of these ribbons are completely inhibited in continuous lighting conditions, they will be regard to be based on external conditions.

\section{Materials and Methods}

Red-eared turtles (body weight; about $10 \mathrm{~g}$ ) were kept in a clear tank $(60 \times 30 \times 36 \mathrm{~cm}$ ) containing water several centimeters in depth. The water was circulated by an air pump and the water temperature was maintained at about $23^{\circ} \mathrm{C}$. Seafood was given two to three times per week at the light period.

The turtles were subjected to a light-dark (LD) cycle with the light on from 0600 to 1800 for at least 10 days. Light was provided indirectly from white fluorescent lamps (National FL 20 SS.W/18). The maximum intensity of the light just above the water surface was 100 lux. At 1000 and 1900, one turtle each was sacrificed for control specimens (Figs. 1 and 2). The other turtles were then subjected to a dark regimen from 1800 for a long period (Fig. 1), or subjected to a light regimen from 0600 for about 2 days (Fig. 2)

At each sampling (Figs. 1 and 2), one or two turtles were decapitated and pithed, and the right eyeball was removed. The left one was also removed for spare specimens. After corneal incisions, the entire eyeball was gently immersed in $2.5 \%$ glutaraldehyde buffered at $\mathrm{pH} 7.3$ with $0.1 \mathrm{M}$ sodium cacodylate at room temperature. The immersion was started within 10 min after decapitation under safelight through a red filter (R-67, Kenko, Tokyo) in the dark period or under about 100 lux in the light period. The eyeball was left in the glutaraldehyde for $1 \mathrm{hr}$ in the dark or under about $100 \mathrm{lux}$, respectively. Then, the retina
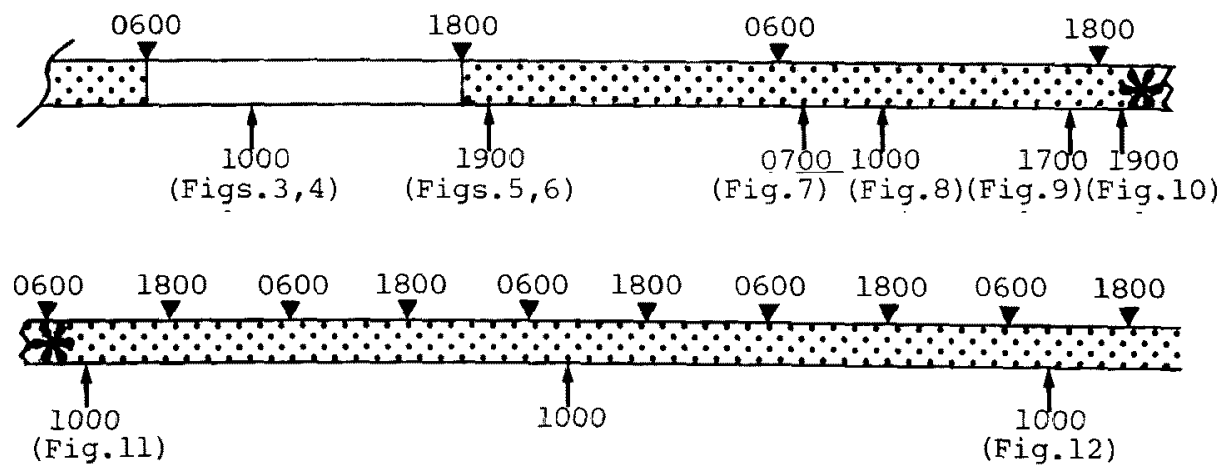

Fig. 1. Dark regimen. 

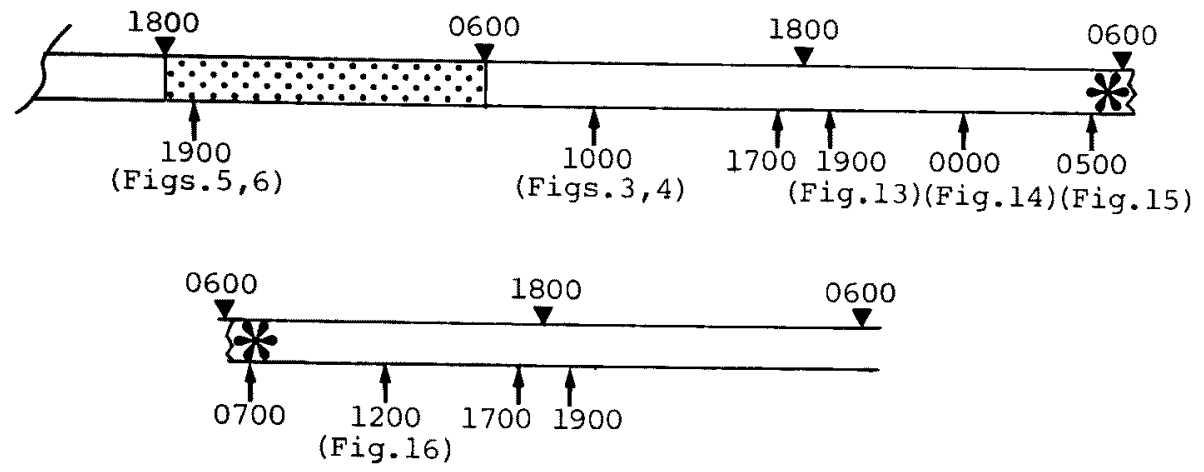

Fig. 2. Light regimen.

around the posterior pole of the eyeball was cut into small pieces, and further fixed in freshly made glutaraldehyde solution for another $1 \mathrm{hr}$. Following a brief wash in cacodylate buffer, the specimens were postifixed in $1 \%$ osmium tetroxide buffered at $\mathrm{pH} 7.3$ with $0.1 \mathrm{M}$ sodium cacodylate for $2 \mathrm{hr}$ at $4^{\circ} \mathrm{C}$. After rinsing several times in distilled water, the specimens were block-stained in $2 \%$ uranyl acetate for $1 \mathrm{hr}$, dehydrated in graded ethanol, and embedded in Epon 812.

Thick sections were cut and stained with toluidine blue, and observed by light microscopy. A couple of blocks were selected for each sampling. Series of 20 to 30 serial thin sections ( $<80 \mathrm{~nm}$ thick) were cut from each block, and picked up on single-hole grids with Formvar film. If necessary, series of 100 to 150 serial thin sections ( $<80 \mathrm{~nm}$ thick) were cut from some blocks, and picked up on $75 / 300$ grids. They were stained with uranyl acetate followed by lead citrate.

When rod cells were identified under electron microscopy (Lasansky 1971; Abe and Yamamoto 1984), their synaptic ribbons were photographed at an original magnification of $\times 15,000, \times 30,000, \times 35,000$ or $\times 42,000$.

For statistical analysis, rod synapses under the following lighting conditions were used: i.e., DD-L, continuous dark (DD) for a long period; DD-10, at 1000 on the first day of DD; DD-19, at 1900 on the first day of DD; LL, continuous light; LD-10, at 1000 in the LD cycle; LD-19, at 1900 in the LD cycle. The numbers of synaptic ribbons per cell were counted in a plane of section. The areas $(S)$, major axes, and perimenters $(P)$ of individual synaptic ribbons were measured on electron micrographs using a computerized image analyzing device, Cosmozone (Nikon, Tokyo). As a morphological index, $4 \pi S / P^{2}$ was calculated. This index of a circle is 1.0 , regardless of its diameter, and a figure with the smaller index is the more markedly deviated from the circle. Wilcoxon's two sample statistics was used to examine the difference of averages. All values are given in terms of mean \pm s.D.

\section{Results}

\section{Diurnal changes}

In $12 \mathrm{hr}$ light-12 hr dark cycle, synaptic ribbons of rod cells formed multilayered ribbon complexes at 1000 , which were composed of parallel arrays of 4 to 8 stick-shaped (plate-shaped, three-dimensionally) ribbons surrounded by a layer of clear synaptic vesicles (Fig. 3). The contour of each ribbon of the 
complex was a little blurred. Only a single layer of clear vesicles was seen in the interspace between adjacent ribbons. Fuzzy substance was observed around the ribbons, suggesting connections between ribbons and clear vesicles (Fig. 4). At 1900 , stick-shaped ribbons disappeared, and their places were taken by aggregates of rounded profiles of ribbon material, although stick-shaped ribbons often persisted just opposite to the arciform density (Fig. 5). Club-shaped ribbons were also observed (Fig. 5, inset). Fuzzy substance was noted between clear vesicles and rounded or club-shaped ribbons (Fig. 6).

\section{Continuous dark (DD)}

After 10 days or more of the LD cycle, turtles were transferred to the dark regimen from 1800, and next day (the first day of DD), one or two turtles were sacrificed at each of the four time-points $(0700,1000,1700$ and 1900) (Fig. 1). At 0700 , no large ribbon complexes could be observed. Instead, single ribbons with a stick-shaped profile were situated close to the presynaptic membrane (Fig. 7). A small number of rounded ribbons or a couple of stick-shaped ribbons arranged in parallel with each other were observed near the presynaptic membrane.

At 1000, although stick-shaped ribbons were sometimes arranged in 2 or 3 parallel layers as shown in Fig. 8 , they were single in most cases. Large ribbon complexes consisting of 7-8 parallel layers, as shown in Fig. 3 (at 1000 of the LD cycle) could never be detected at 1000 in $\mathrm{DD}$, neither could be rounded ribbons. The contour of stick-shaped ribbons was always sharp at 1000 .

At 1700 , single stick-shaped ribbons were observed near the presynaptic membrane (Fig. 9). Sometimes a small number of rounded ribbons and a couple of parallel stick-shaped ones were also seen near the stick-shaped ribbons.

At 1900, neither large ribbon complexes nor aggregates of rounded ribbons could be recognized. Instead, stick-shaped ribbons were situated singly or sometimes arranged in a few parallel layers (Fig. 10). They showed sharp contours.

As continuing the dark regimen, one turtle each was sacrificed at 1000 on the second, fourth, and the sixth day (Fig. 1). Figs. 11 and 12 show ribbon complexes composed of a small number of stick-shaped ribbons obtained at 1000 on the second and sixth days, respectively. At 1000 on the fourth day, no ribbon complexes, but single stick-shaped ribbons, could be observed.

In $\mathrm{DD}$, the rod spherule contained a larger amount of coated vesicles than in the LD cycle.

\section{Continuous light (LL)}

After 10 days or more of the LD cycle, turtles were transferred to the light regimen from 0600 (Fig. 2). One turtle each was sacrificed at time-points of 1700 , and 1900. Next day, one turtle each was also sampled at 0000, 0500, 0700, 1200, 1700 and 1900. Neither stick-shaped ribbons nor aggregates of rounded ribbons could be observed in any of these specimens. Instead, aggregates of irregular 
ribbon fragments were noted, as shown in Figs. 13 (at 1900), 14 (at 0000), 15 (at 0500), and 16 (at 1200). None of the ribbons in this light regimen showed sharp contours regardless of their shape.

\section{Statistical analysis}

The average number of synaptic ribbons per cell in LL was $11.2 \pm 3.2$, which was significantly larger $(p<0.01)$ than in DD (DD-L, DD-10, DD-19). This number in LL was also significantly larger $(p<0.05)$ than in LD (LD-10, LD-19) (Table 1). The average number of ribbons in LD-19 (7.5 \pm 3.0$)$ was significantly larger $(p<0.01)$ than in DD.

The areas of individual synaptic ribbons in DD-L $\left(0.041 \pm 0.032 \mu \mathrm{m}^{2}\right)$ were significantly larger $(p<0.01)$ than those in any others. The areas in DD-19 $\left(0.027 \pm 0.030 \mu \mathrm{m}^{2}\right)$ were significantly larger than those in $\mathrm{LL}(p<0.01)$ and LD-10 $(p<0.05)$. Those in DD-10 $\left(0.023 \pm 0.015 \mu \mathrm{m}^{2}\right)$ were significantly larger $(p<0.01)$ than those in LL and LD-10. Those in LD-19 $\left(0.018 \pm 0.010 \mu \mathrm{m}^{2}\right)$ were significantly larger $(p<0.01)$ than in LL. Note those in DD were larger than those in LL and LD-10. In DD, DD-L showed larger areas than DD-10 and DD-19, but no significancy was obtained between DD-10 and DD-19.

The major axes of individual ribbons in DD-L $(0.65 \pm 0.39 \mu \mathrm{m})$ were significantly larger than those in DD-19 $(p<0.05)$ and those in any others $(p<$ 0.01). Those in DD-19 $(0.46 \pm 0.34 \mu \mathrm{m})$ and DD-10 $(0.38 \pm 0.21 \mu \mathrm{m})$ were significantly larger $(p<0.01)$ than those in LL, LD-10, and LD-19. Those in LD-10 $(0.28 \pm 0.16 \mu \mathrm{m})$ were significantly larger than those in LL $(p<0.05)$ and LD-19 $(p<0.01)$. Note DD showed large axes than LL, LD-10, and LD-19, which received continuous or cyclic illumination. In DD, DD-L had larger axes than

TABLE 1. Morphometrical data on synaptic ribbons of the turtle rod cells under various lighting conditions

\begin{tabular}{ccccc}
$\begin{array}{c}\text { Lighting } \\
\text { condition }\end{array}$ & $\begin{array}{c}\text { Number of } \\
\text { ribbons/cell }\end{array}$ & $\begin{array}{c}\text { Area }(S) \\
\left(\mu \mathrm{m}^{2}\right)\end{array}$ & $\begin{array}{c}\text { Major axis } \\
(\mu \mathrm{m})\end{array}$ & $\begin{array}{c}\text { Morphological } \\
\text { index } \\
\left(4 \pi S / P^{2}\right)\end{array}$ \\
\hline DD-L & $3.0 \pm 1.2$ & $0.041 \pm 0.032$ & $0.65 \pm 0.39$ & $0.33 \pm 0.18$ \\
$(n=32)$ & $(n=32)$ & $(n=32)$ \\
DD-10 & $(n=11)$ & $0.023 \pm 0.015$ & $0.38 \pm 0.21$ & $0.46 \pm 0.18$ \\
& $(n=1.9$ & $(n=42)$ & $(n=42)$ & $(n=42)$ \\
DD-19 & $3.5 \pm 1.3$ & $0.027 \pm 0.030$ & $0.46 \pm 0.34$ & $0.38 \pm 0.18$ \\
& $(n=12)$ & $(n=41)$ & $(n=41)$ & $(n=41)$ \\
LL & $11.2 \pm 3.2$ & $0.014 \pm 0.012$ & $0.21 \pm 0.11$ & $0.52 \pm 0.17$ \\
& $(n=10)$ & $(n=105)$ & $(n=105)$ & $(n=105)$ \\
LD-10 & $5.5 \pm 4.1$ & $0.015 \pm 0.010$ & $0.28 \pm 0.16$ & $0.49 \pm 0.19$ \\
& $(n=10)$ & $(n=54)$ & $(n=54)$ & $(n=54)$ \\
LD-19 & $7.5 \pm 3.0$ & $0.018 \pm 0.010$ & $0.19 \pm 0.07$ & $0.79 \pm 0.15$ \\
& $(n=12)$ & $(n=85)$ & $(n=85)$ & $(n=85)$ \\
& & &
\end{tabular}

$P$, perimeter. Values are given in terms of mean \pm S.D. 
DD-10 and DD-19, but no significancy was obtained between DD-10 and DD-19.

The morphological indices $\left(4 \pi S / P^{2}\right)$ of individual synaptic ribbons in LD-19 $(0.79 \pm 0.15)$ were significantly larger $(p<0.01)$ than those in any others. Those in LL $(0.52 \pm 0.17)$ and LD-10 $(0.49 \pm 0.19)$ were significantly larger $(p<0.01)$ than those in DD-L and DD-19. In DD, DD-10 $(0.46 \pm 0.18)$ showed significantly larger indices $(p<0.01)$ than DD-L. Note synaptic ribbons in LD-19 were the most rounded in shape. The indices in DD were smaller than LL, LD-10, and LD-19, which received continuous or cyclic illumination. DD-L had the smallest index.

\section{Discussion}

As reported in our previous paper (Abe and Yamamoto 1984), synaptic ribbons of the rod cells of the turtle showed marked diurnal changes in structure. After 10 days of the LD cycle, the ribbons form multilayered ribbon complexes at 1000. At 1900, aggregates of rounded ribbons are predominant. Unique rounded shapes in LD-19 were confirmed by the largest morphological index and by the smallest major axis. The numbers of parallel arrays of ribbons of the ribbon complexes are maximal at 1000 to 1200 , though the contour of each stick-shaped ribbon is a little blurred at these time points.

When the turtles are transferred to the dark regimen, most synaptic ribbons of the rod cells show single stick-shaped profiles at 0700, 1000, 1700 and 1900 . Only a small number of ribbons are arranged in parallel to each other. At 1700 , no aggregates of irregular ribbon fragments can be observed, nor aggregates of rounded ribbons at 1900 . The ribbons keep the single stick form or parallel arrangement of a few ribbon profiles for the following days. Thus, the diurnal changes in LD no longer take place when the animals are transferred to DD. In $\mathrm{DD}$, the ribbons remain in a state of 0000 or 0500 of the LD cycle. Interestingly, in DD, ribbons in DD-L were longer and larger than those in DD-10 and in DD-19. Prolonged dark adaptation may have some effects on the elongation of synaptic ribbons.

In the fish retina, the number of synaptic ridges containing a single stickshaped ribbon shows circadian rhythmicity when the fish are transferred to the constant dark (Wagner 1975). In the turtle retina, the authors are skeptical over the contribution of internal rhythm to the diurnal changes in the structure of the synaptic ribbon.

Besharse and Iuvone (1983) reported circadian fluctuation of $\mathrm{N}$ acetyltransferase activity in the Xenopus retina and suggested circadian rhythmicity of the metabolism of retinal melatonin in the eyecup in DD. We cannot comment on melatonin levels in the turtle retina.

When the turtles were transferred to the light regimen from 0600, aggregates of irregular, fragmental ribbons are observed at any time from 1700 to the next day. Individual ribbons in LL were smaller and shorter, and the number of ribbons per cell was markedly larger than in DD. These figures are similar to 
those at 1700 in the LD cycle as reported previously (Abe and Yamamoto 1984). This finding suggests that, in LL, the diurnal changes stop and that the ribbons remain in the state of 1700 of LD for a long period. Multilayered ribbon complexes are formed at the light period in the LD cycle, but LL is not effective to promote the ribbon complex formation. Cyclic light-dark illumination is necessary to form multilayered ribbon complexes and rounded ribbons.

\section{Acknowledgments}

We thank Dr. Seiki Kataoka and Dr. Jack Rosenbluth for their helpful suggestions. They also thank Mr. Hiroo Iwasa for his tecnical assistance and Miss Kumi Miura for typing the manuscript.

\section{References}

1) Abe, H. \& Yamamoto, T.Y. (1984) Diurnal changes in synaptic ribbons of rod cells of the turtle. J. Ultrastruct. Res., 86, 246-251.

2) Besharse, J.C. \& Iuvone, P.M. (1983) Circadian clock in Xenopus eye controlling retinal serotonin $N$-acetyltransferase. Nature (Lond.), 305, 133-135.

3) Lasansky, A. (1971) Synaptic organization of cone cells in the turtle retina. Phil. Trans. royal Soc. London, Ser. B, 262, 365-381.

4) Matsushima, S., Morisawa, Y., Aida, I. \& Abe, K. (1983) Circadian variations in pinealocytes of the chinese hamster, Cricetulus griseus. A quantitative electronmicroscopic study. Cell Tissue Res., 228, 231-244.

5) McArdle, C.B., Dowling, J.E. \& Masland, R.H. (1977) Development of outer segments and synapses in the rabbit retina. $J$. comp. Neurol., 175, 253-274.

6) McNulty, J.A. (1981) Synaptic ribbons in the pineal organ of the goldfish: circadian rhythmicity and the effects of constant light and constant darkness. Cell Tissue Res., 215, 491-497.

7) Olney, J.W. (1968) An electron microscopic study of synapse formation, receptor outer segment development, and other aspects of developing mouse reitna. Invest. Ophthal., 7, 250-268.

8) Sjöstrand, F.S. (1958) Ultrastructure of retinal rod synapses of the guinea pig eye as revealed by three-dimensional reconstructions from serial sections. J. Ultrastruct. Res., 2, 122-170.

9) Spadaro, A., de Simone, I. \& Puzzolo, D. (1978) Ultrastructural data and chronobiological patterns of the synaptic ribbons in the outer plexiform layer in the retina of albino rats. Acta anat., 102, 365-373.

10) Vollrath, L. (1981) The Pineal Organ. In: Handbuch der mikroskopishen Anatomie des Menschen, edited by A. Oksche \& L. Vollrath, Vol. VI/7. Springer-Verlag, Berlin, pp. 146-158.

11) Wagner, H.-J. (1973) Darkness-induced reduction of the number of synaptic ribbons in fish retina. Nature new Biol., 246, 53-55.

12) Wagner, H.-J. (1975) Quantitative changes of synaptic ribbons in the cone pedicles of Nannacara : Light dependent of governed by a circadian rhythm? In: Vision in Fishes, New Approaches in Research, edited by M.A. Ali, Plenum Press, New York, pp. $679-686$. 
Fig. 3. Multilayered ribbon complex at 1000 in the light-dark (LD) cycle. $\times 44,800$.

Fig. 4. Enlarged view of multilayered ribbon complex at 1000 in LD cycle. $\times 113,000$.

Fig. 5. Aggregates of rounded ribbons at 1900 in LD cycle. $\times 45,100$. Clubshaped ribbons were also seen. $\times 38,900$ (inset).

Fig. 6. Enlarged view of rounded ribbons at 1900 in LD cycle. $\times 113,000$. 


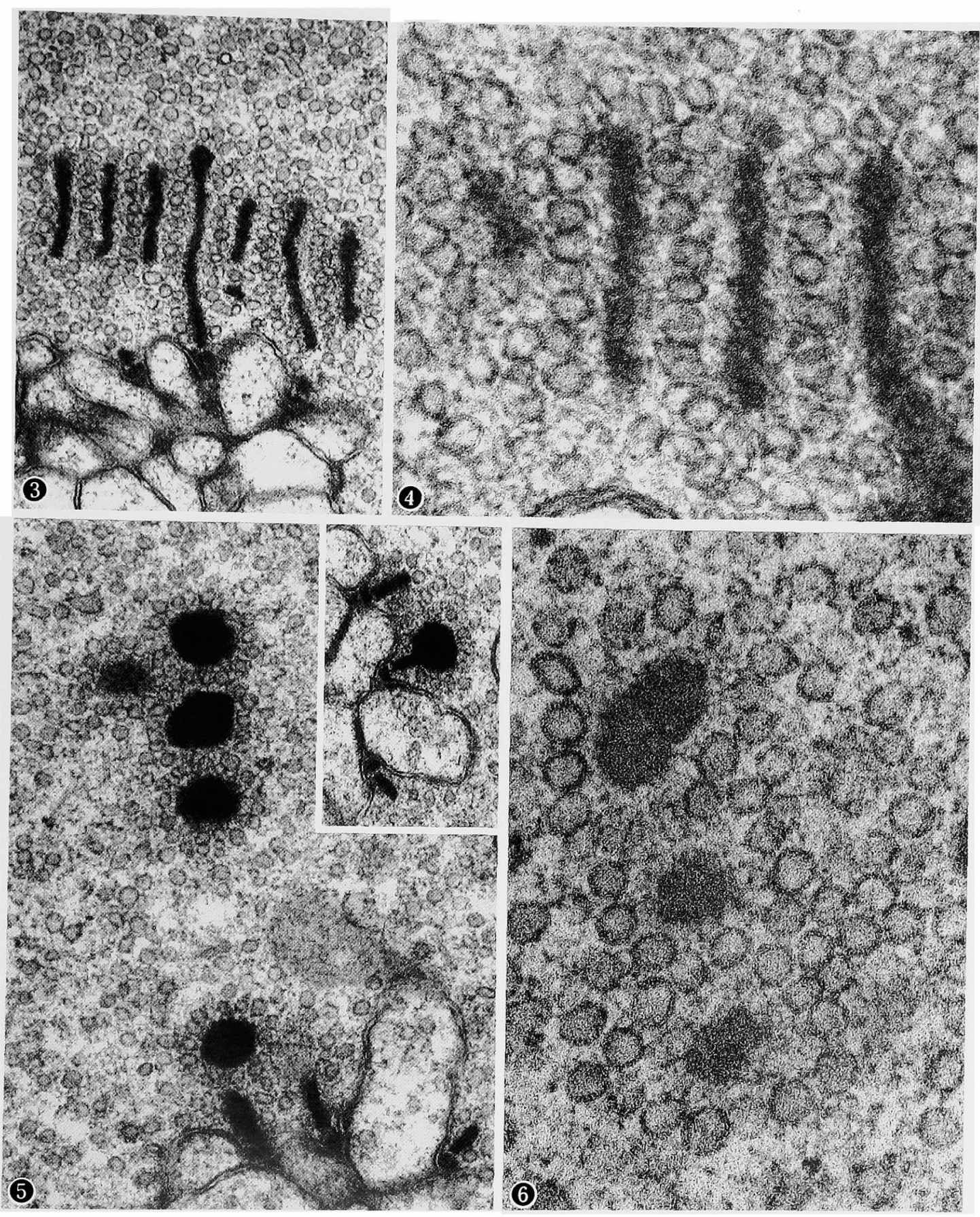


Figs. 7-12 show ribbons in the continuous dark (DD).

Fig. 7. At 0700 on the first day of DD. $\times 34,500$.

Fig. 8. At 1000 on the first day of DD. $\times 33,800$.

Fig. 9. At 1700 on the first day of DD. $\times 34,500$.

Fig. 10. At 1900 on the first day of DD, $\times 34,500$.

Fig. 11. At 1000 on the second day of DD. $\times 33,800$.

Fig. 12. At 1000 on the sixth day of DD. $\times 34,500$. 

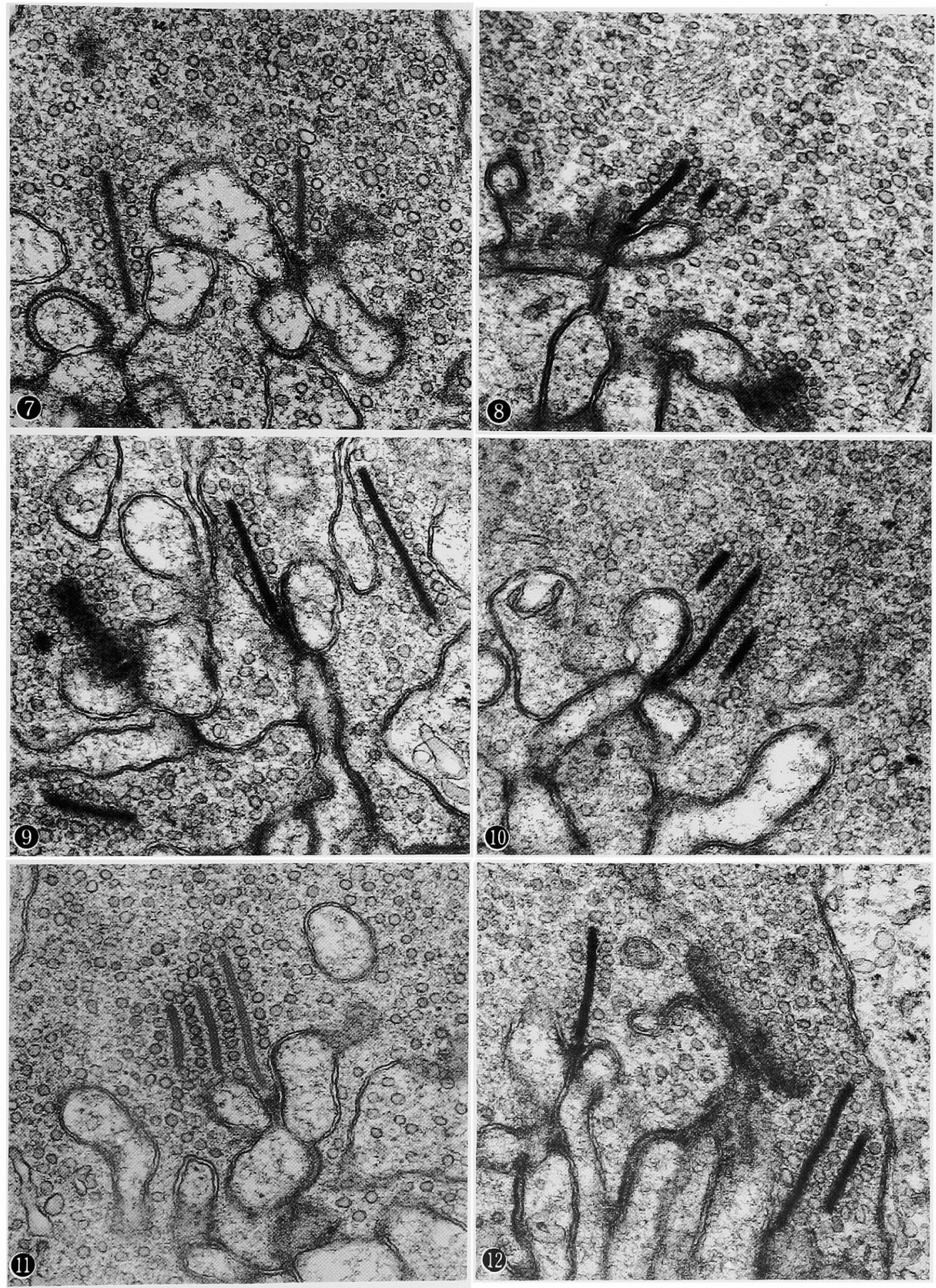
Figs. 13-16 show ribons in the continuous light (LL).

Fig. 13. At 1900 in LL. $\times 59,400$.

Fig. 14. At 0000 in LL. $\times 59,900$.

Fig. 15. At 0500 in LL. $\times 59,400$.

Fig. 16. At 1200 in LL. $\times 60,200$. 

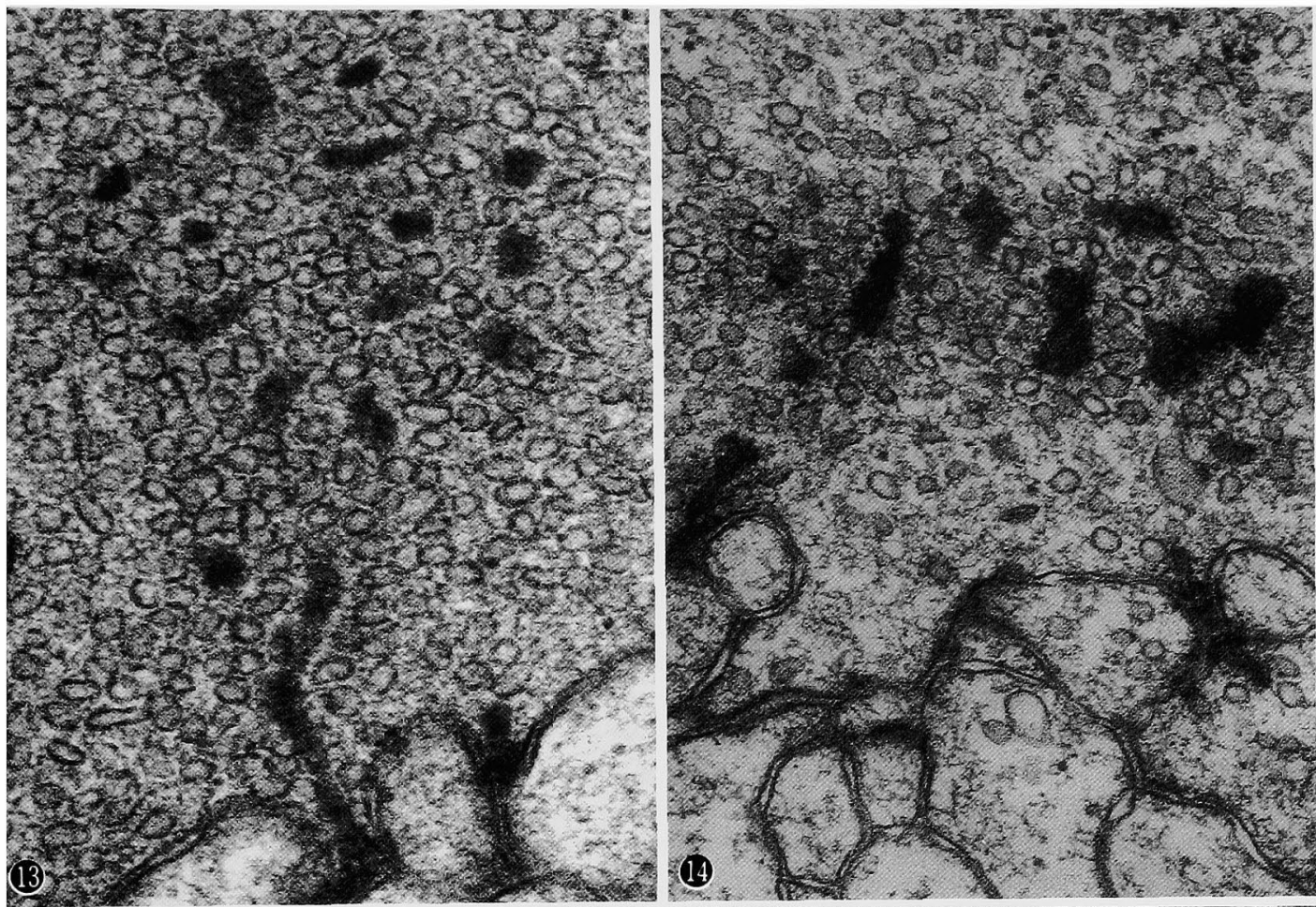

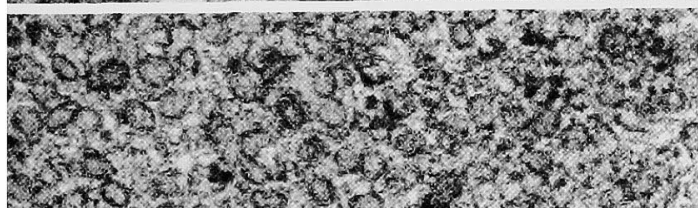

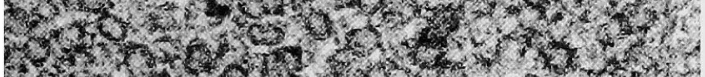
1.7. 71 (3)

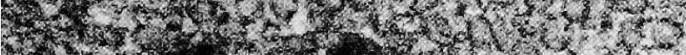

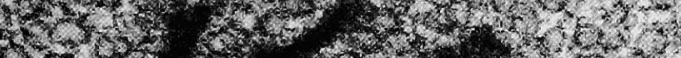

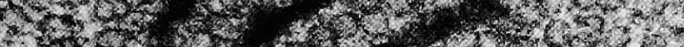
2.

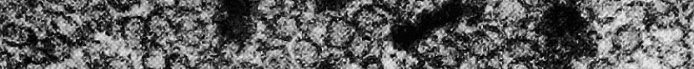

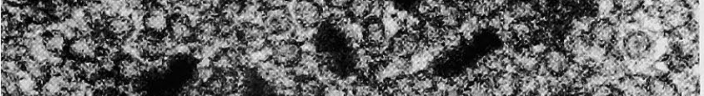
1.7.

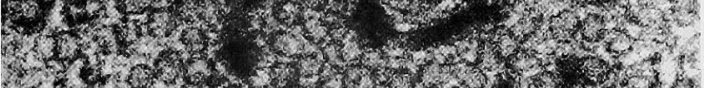

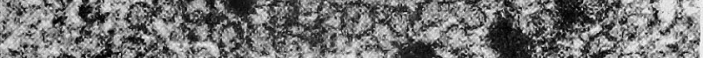

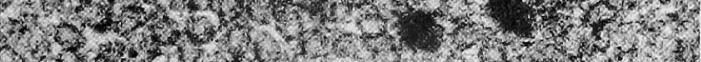

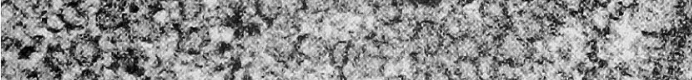

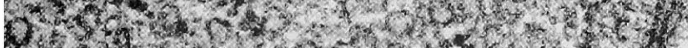

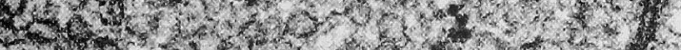

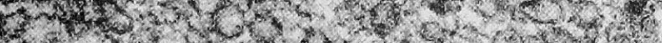

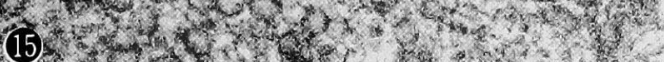

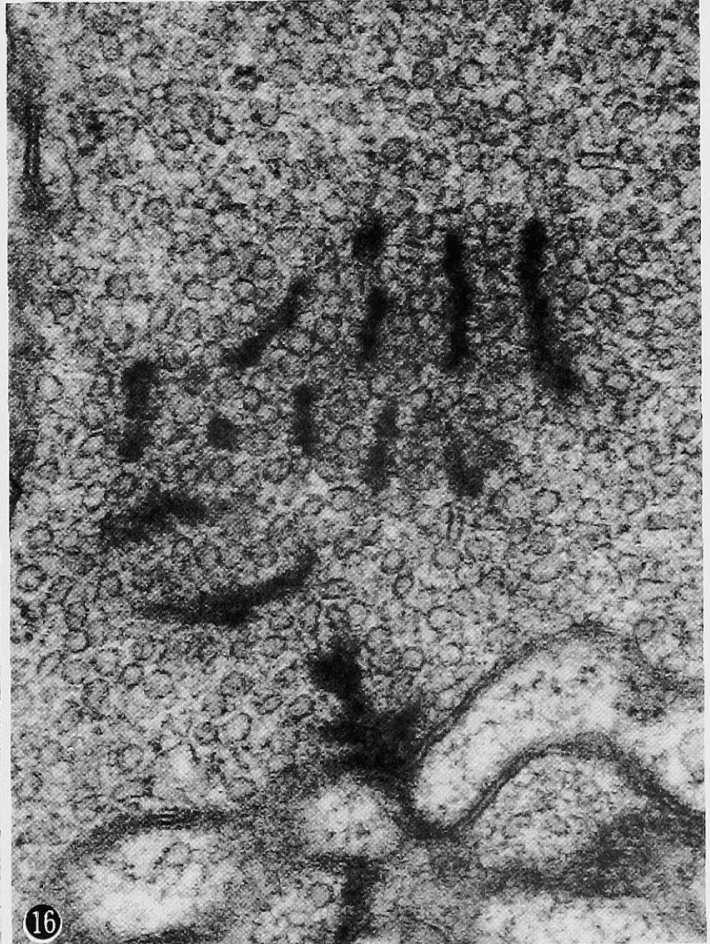

